\title{
Electrical properties of naphthyl polyenes
}

\author{
SEEMA SEN, PRABIR PAL and T N MISRA* \\ Spectroscopy Department, Indian Association for the Cultivation of Science, Calcutta 700032 , \\ India
}

MS received 11 August 1993; revised 20 December 1993

\begin{abstract}
Dark and photoconductive properties of $\alpha-\omega$-di-2-naphthyl polyenes were studied at different applied voltages and temperatures. These studies indicate that a single dominant trapping level is involved in both conduction processes. From this study some transport parameters are evaluated. Dependence of photocurrent on excitation light intensity indicates that the carrier generation process is a one-photon process and is trap-limited. The photocurrent decay study indicates that most of the photocarriers decay exponentially with time. Trap depths are calculated from the temperature dependence of decay constant and is found to be the same as that obtained from thermally stimulated current. The dependence of photocurrent on wavelength indicates two mechanisms of photocarrier generation process: one is excitonic and the other is intrinsic electron-hole pair production.
\end{abstract}

Keywords. Dark and photoconduction; polyenes; organic semiconductor; organic photoconductor; trap; decay kinetics.

\section{Introduction}

Photoconductive properties of carotenoid polyenes have been investigated extensively (Hoshino and Tateishi 1979; Pal et al 1988; Pal and Misra 1989, 1993). The highly conjugated $\pi$-electronic structure of these molecules results in their semiconducting and photoconductive properties in the solid state. We have now extended such studies to a class of synthetic organic materials with polyene structure. Voltage and temperature dependence of current, decay kinetics and variation of photocurrent with excitation light intensity and wavelength of $\alpha$ - $\omega$-di-2-naphthyl polyenes of the type $\mathrm{R}-(\mathrm{CH}=\mathrm{CH})_{n}-\mathrm{R}$ (where $\mathrm{R}$ stands for the naphthyl group and $n=3,5$ ) have been studied in sandwich cell configuration. These studies provide information about dark and photocarrier generation and transport mechanisms in these materials and elucidates the effect of chain length on their electrical properties. In this paper we report our results.

\section{Experimental}

$\alpha-\omega$-Di-2-naphthyl polyenes were obtained as a gift from Prof. Nakagawa (Osaka University, Japan), who synthesized these materials (Yasuhara et al 1972). Their convention of denoting these naphthyl polyenes as $I_{n}^{\prime}$ is also used by us. The molecular structure of $\alpha-\omega$-naphthyl polyenes is shown in the inset of figure 4 . The melting point of $I_{3}^{\prime}$ is $251-262^{\circ} \mathrm{C}$ and that of $I_{5}^{\prime}$ is $268-269^{\circ} \mathrm{C}$. Conductivity measurements were made in a sandwich cell with stainless steel and $\mathrm{SnO}_{2}$-coated glass electrodes separated by a Teflon spacer of $0.005 \mathrm{~cm}$. Finely powdered samples sandwiched between the electrodes were kept at a moderate pressure of $4400 \mathrm{~g} / \mathrm{cm}^{2}$ by spring

\footnotetext{
* For correspondence
} 
clips. The cell was then placed in a suitably designed conductivity chamber (Mallick et al 1979) and was subjected to heat-cool cycles in vacuum to remove any adsorbed gas on the crystallite surfaces. Other experimental methodology was the same as described earlier (Pal and Misra 1989).

\section{Results and discussion}

\subsection{Variation of current with voltage}

Generally for ohmic contact the current-voltage relation in organic semiconductors is ohmic in nature up to a certain voltage and then it becomes space-charge-limited. The $I-V$ plots for both $I_{3}^{\prime}$ and $I_{5}^{\prime}$ are ohmic (within our experimental range of applied fields) for dark currents but for photocurrents these are ohmic up to a certain field, above which they show square law dependence on voltage. The cross-over fields from ohmic to space-charge region are $1.14 \times 10^{3} \mathrm{~V} / \mathrm{cm}$ and $1.52 \times 10^{3} \mathrm{~V} / \mathrm{cm}$ for $I_{3}^{\prime}$ and $I_{5}^{\prime}$ respectively. Since every real solid contains traps as a consequence of structural imperfections or presence of impurities the quadratic dependence of current on voltage may be due to the presence of a single discrete trapping level or exponential trap distribution (Rose 1955; Lampert 1964).

For exponential trap distribution it has been shown that the current will be quadratic at $T=T_{c}$. Hence the quadratic relation of current on voltage does not favour any particular type of trap distribution. A plot of $\log _{10} I$ vs $1 / T$ should yield a straight line for both the cases. For a single discrete trapping level, however, the slope of such a plot is independent of applied voltage, whereas for exponential trap distribution the slope is a voltage-dependent quantity. The $\log _{10} T$ vs $1 / T$ plots at different voltages are shown in figure 1 for $I_{3}^{\prime}$ and $I_{5}^{\prime}$. It is found that for both the samples the slopes of photocurrents are voltage-independent, which shows evidence of the involvement of a single discrete dominant trapping level for photoconduction.

\subsection{Evaluation of some transport parameters}

The transition voltage from ohmic to space-charge region is given by Rose (1955) and Lampert (1964):

$$
\begin{aligned}
V_{t} & =(8 / 9)\left(q d^{2} n_{0}\right) / \varepsilon_{0} \theta \\
& \left.=(8 / 9)\left(d^{2} / \mu_{e} \tau \varepsilon_{0}\right)=(8 / 9) d^{2} \sigma / \mu_{e} \varepsilon \varepsilon_{0}\right)
\end{aligned}
$$

where $\sigma$ is the room temperature conductivity at the cross-over voltage, $\tau=\varepsilon / \sigma$ is the relaxation time, and the other notations have their usual meanings.

Since $\varepsilon$ values of $I_{3}^{\prime}$ and $I_{5}^{\prime}$ are not known we have taken the $\varepsilon$ value for naphthalene, which is 2.5 (Weast et al 1986), and using this value the effective drift mobility $\mu_{e}$ has been calculated. The results are summarized in table 1 . The low value of $\mu_{e}$ suggests that the conduction mechanism is a hopping process. Now $V_{t}$ would be high if $\theta$ (i.e. the ratio of free to trapped charge) is low. So we can conclude that for dark conduction in both $I_{3}^{\prime}$ and $I_{5}^{\prime}, \theta$ is very low. Indeed a large number of trapped carriers are photoexcited at higher fields giving a large photocurrent. For dark conduction ohmic 


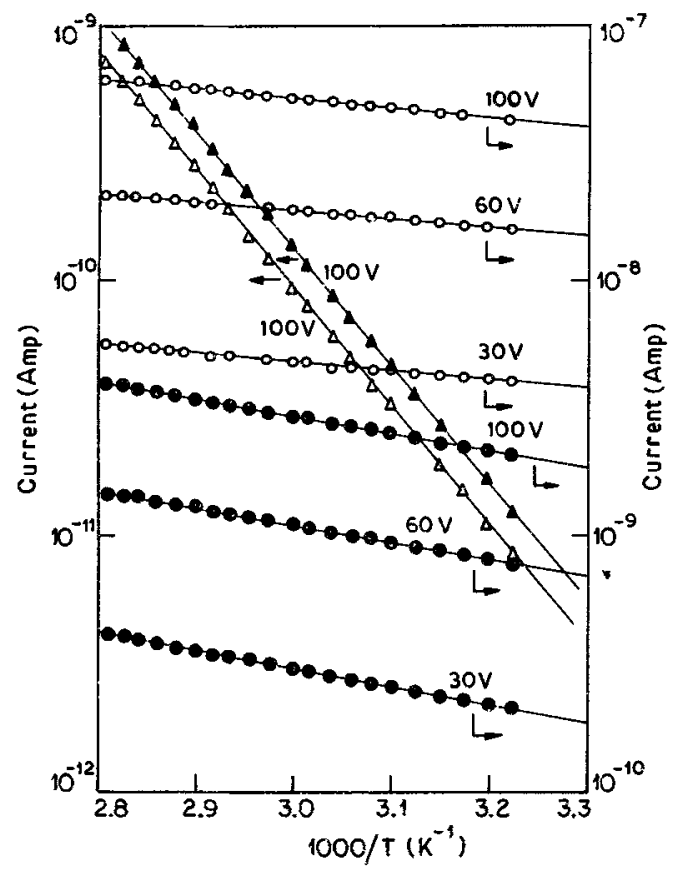

Figure 1. The plots of $\log I$ vs $1000 / T$ for $I_{3}^{\prime}$ and $I_{5}^{\prime}$ at various applied voltages. $-\triangle-\triangle-\triangle-$ represents dark and $-\mathrm{O}-\mathrm{O}-\mathrm{O}$ - represents photoconductivity in $I_{3}^{\prime}$ and $-\mathbf{\Delta}-\mathbf{\Delta}-\mathbf{\Delta}-$ dark and $-1-$ photoconductivity in $I_{5}^{\prime}$.

Table 1. Values of some transport parameters in $I_{3}^{\prime}$ and $r_{5}^{\prime}$.

\begin{tabular}{lccc}
\hline Material & $\begin{array}{c}\text { Nature of } \\
\text { conductivity }\end{array}$ & $\begin{array}{c}\mu_{e} \text { calculated } \\
\text { from }(2) \\
\left(\mathrm{cm}^{2} \mathrm{~V}^{-1} \mathrm{sec}^{-1}\right)\end{array}$ & $\begin{array}{c}n_{0} / \theta \text { calculated } \\
\text { from }(1) \\
\left(\mathrm{cm}^{-3} \text { at } 300 \mathrm{~K}\right)\end{array}$ \\
\hline$I_{3}^{\prime}$ & Photo & $6.38 \times 10^{-6}$ & $3.55 \times 10^{11}$ \\
$I_{5}^{\prime}$ & Photo & $3.31 \times 10^{-6}$ & $3.32 \times 10^{11}$ \\
\hline
\end{tabular}

to space-charge transitions do not occur even at a field of $2 \times 10^{4} \mathrm{~V} / \mathrm{cm}$ but for photoconduction the space-charge region begins at a much lower field.

\subsection{Temperature dependence of dark and photoconduction}

The temperature dependence of dark current is given by

$$
I_{\mathrm{d}}=I_{\mathrm{od}} \exp \left(-E_{\mathrm{d}} / 2 k T\right)
$$

In some materials both the dominant trapping levels (for electrons and holes) contribute equally and the material behaves as a non-extrinsic semiconductor (Roberts and Schmidt 1969). On light excitation, if different trap levels are populated and contribute significantly to photoconduction the extrinsic nature is retained. The photocurrent 
under this condition is given by

$$
I_{\mathrm{ph}}=I_{\mathrm{op}} \exp \left(E_{\mathrm{ph}}^{\prime} / K T\right) \text {. }
$$

In figure 1 the temperature dependence of dark and photoconduction for both the materials are shown. The value of dark activation energy $E_{\mathrm{d}}$ is equal to $1.85 \mathrm{eV}$ for both materials and photoactivation energy $E_{\mathrm{ph}}^{\prime}$ for $I_{3}^{\prime}$ and $I_{s}^{\prime}$ are $0.066 \mathrm{eV}$ and $0.140 \mathrm{eV}$ respectively. Such a low photoactivation energy is quite general for organic materials and is thought to be the exciton dissociation energy. The observed difference of $E_{\mathrm{ph}}^{\prime}$ values in the two materials may be due to different temperature dependence of photodetrapping and/or recombination rates in them.

The calculated $E_{\mathrm{d}}$ and $E_{\mathrm{ph}}^{\prime}$ are independent of applied fields, as expected for semiconductors with single discrete trapping levels. From these studies of $I$ vs $V$ and $\log I$ vs $1 / T$ it is seen that for any particular voltage dark current is almost the same for both the samples while photocurrent is about two orders less in $I_{5}^{\prime}$ than in $I_{3}^{\prime}$. Thus we may conclude that increase of chain length does not play any significant role in dark conduction but photoconduction is chain length-dependent. Indeed chain length affects the spectroscopic properties of the molecules. Photoactivation energy in $I_{3}^{\prime}$ is lower than in $I_{5}^{\prime}$.

\subsection{Excitation light intensity dependence of photocurrent}

In both organic and inorganic photoconductors the light intensity $I_{B}$ and the photocurrent $I_{\mathrm{ph}}$ are related by the relation

$$
I_{\mathrm{ph}}=I_{\mathrm{B}}^{\gamma}
$$

where $\gamma$ is a characteristic of the photoconducting material. So a plot of $\log I_{\mathrm{ph}}$ vs $\log I_{\mathrm{B}}$ will yield a straight line.

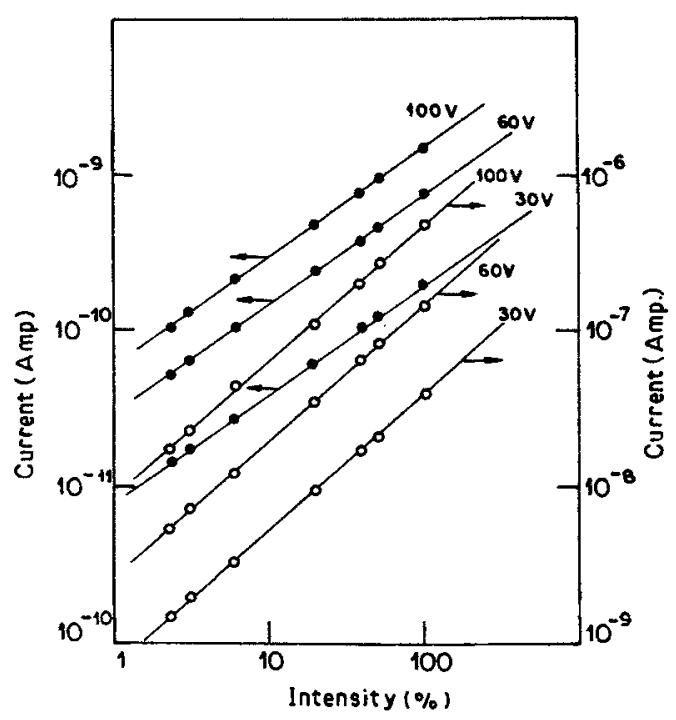

Figure 2. Photocurrent vs light intensity dependence in logarithmic scale at different applied voltages. $-\mathrm{O}-\mathrm{O}-\mathrm{O}-, \mathrm{I}_{3}$; 
To study the excitation light intensity dependence on photoconductor the sandwich cell was excited through the positive electrode. It is found (figure 2) that the plots yield $\gamma=0.87$ for $I_{3}^{\prime}$ and $\gamma=0.70$ for $I_{5}^{\prime}$ and values of $\gamma$ for both $I_{3}^{\prime}$ and $I_{5}^{\prime}$ are independent of applied voltage. This sublinear intensity dependence is the result of cooperation of traps and recombination centres. From the curve it is evident that the photoconduction is a one-photon process for all applied voltages.

\subsection{Photoconductivity decay}

Direct recombination of electron-hole pairs and recombination via shallow traps give first-order decay of photoconductivity. $I_{\mathrm{ph}}$ in this case follows the relation

$$
I_{\mathrm{ph}}=I_{0} \exp \left(-t / \tau_{0}\right)
$$

where $I_{0}$ is the initial photocurrent at $t=0$ and $\tau_{0}$ is the mean decay time. Secondorder decay occurs when retrapping of carriers freed from the traps is significant, and the corresponding relation (Rose 1969) between $I_{\mathrm{ph}}$ and $t$ is

$$
1 / I_{\mathrm{ph}}=k t+C
$$

where $k$ and $C$ are positive constants.

In naphthyl polyenes it is found that most of the photocarriers decay by first-order process. The semilogarithmic plot of $I_{\mathrm{ph}}$ with time for $I_{3}^{\prime}$ and $I_{5}^{\prime}$ is shown in figure 3. The estimated $\tau_{0}$ values are 0.417 and $21.50 \mathrm{msec}$ for $I_{3}^{\prime}$ and $I_{5}^{\prime}$ respectively. Now the observed decay time $\tau_{0}$ can be related to the carrier lifetime $\tau$ if the electron and hole population densities in the traps and the lifetime of free electrons and holes are known.

The semilogarithmic plot of $\tau_{0}$ with $1 / T$ (inset of figure 3 ) yields a straight line whose slope represents the trap depth. The calculated values of trap depths are $0.063 \mathrm{eV}$ and $0.145 \mathrm{eV}$ for $I_{3}^{\prime}$ and $I_{5}^{\prime}$ respectively, which are very close to the trap depths obtained from photocurrent temperature studies. Thus we conclude that photocarrier generation is dominant shallow trap-limited.

\subsection{Photoconduction action spectra}

Generally in organic semiconductors the action spectra are similar to absorption spectra. On the other hand, in some organic semiconductors there is a reversal and minima of absorption spectra may coincide with maxima in photoconduction spectra (Ghosh et al 1974; Glenis et al 1984). Figure 4 shows the wavelength dependence of photocurrent and thin film absorption spectrum in $I_{3}^{\prime}$. It is found that there is no change in the response of $I_{\mathrm{ph}}$ on negative and positive electrode illumination. In the spectral range $320-420 \mathrm{~nm}$ the action spectrum of $I_{3}^{\prime}$ resembles the absorption spectrum. However, in the region between 421 and $600 \mathrm{~nm}$ such a similarity disappears. Similar behaviour is observed for $I_{5}^{\prime}$. The similarity between action and absorption spectra within a certain wavelength range suggests that the primary step leading to carrier generation is excitation of levels which corresponds to those in the absorption spectra. The carriers are generated by exciton dissociation.

For both the naphthyl polyenes there is a peak in the long-wavelength region, i.e. $421-600 \mathrm{~nm}$ for $I_{3}^{\prime}$ and $481-620 \mathrm{~nm}$ for $I_{5}^{\prime}$. The structural features of these bands are 


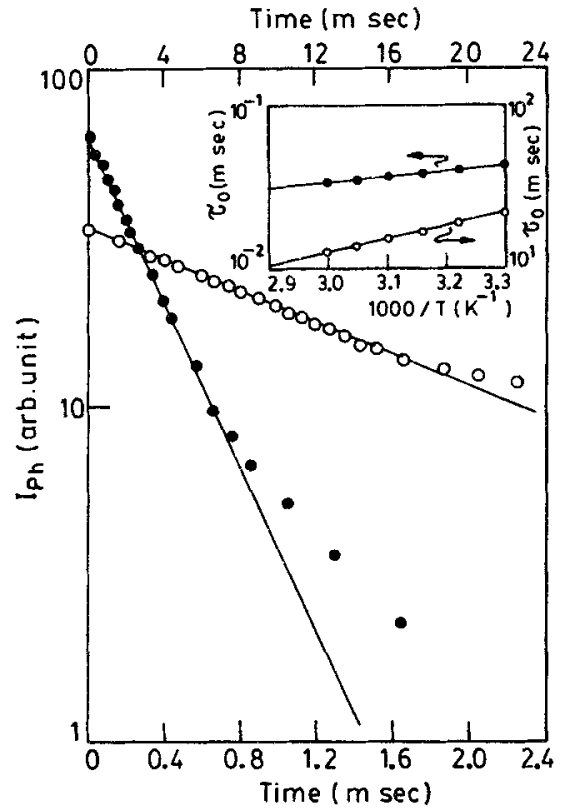

Figure 3. The semilogarithmic plot of $l_{\mathrm{ph}}$ with time for $I_{3}^{\prime}$ and $I_{5}^{\prime} .-1-I_{3}^{\prime}$; $-O-O-O-, I_{5}^{\prime}$. Inset: The semilogarithmic plot of decay constant $\tau$ with $1000 / T$ for $I_{3}^{\prime}$ and $I_{5}^{\prime} ;-\bullet-\bullet-, I_{3}^{\prime} ;-O-O-O-, I_{s}^{\prime}$.

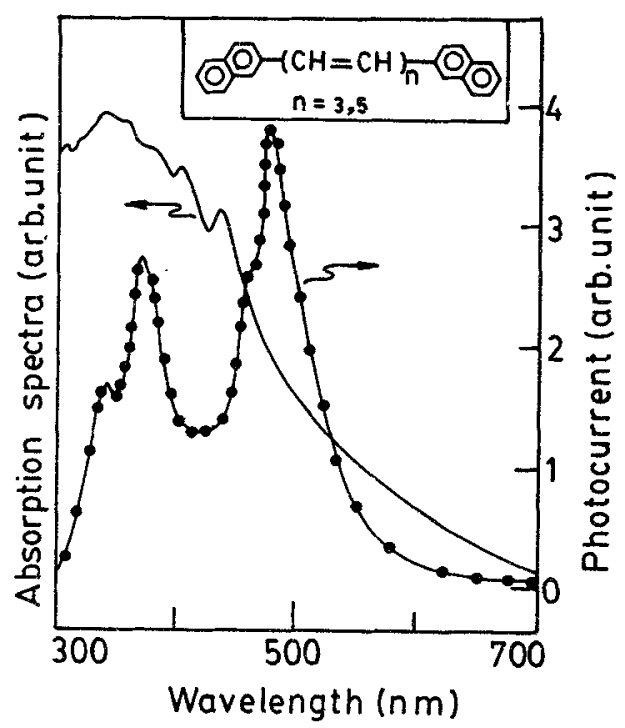

Figure 4. The wavelength dependence of photocurrent and absorption spectrum in solid film for $I_{3}^{\prime}$. Inset: Molecular structure of $\alpha$ - $\omega$-di-2-naphthyl polyenes. 
distinctly different from those in the corresponding absorption spectra, Such a long-wavelength peak was found in some other polyene semiconductors ( $\mathrm{Pal}$ and Misra 1989,1993 ). If we extrapolate the long-wavelength tails of the action spectra they intersect the abscissa at $610 \mathrm{~nm}(2.03 \mathrm{eV})$ for $I_{3}^{\prime}$ and at $660 \mathrm{~nm}(1.88 \mathrm{eV})$ for $I_{5}^{\prime}$. These values agree fairly well with the corresponding thermal activation energies for dark conduction as obtained from $\log I$ vs $1 / T$ plot. This suggests that in the two naphthyl polyenes the intrinsic mechanism of carrier generation (direct electron-hole pair production) is operative both in dark and photoconduction. A similar observation has been reported for many natural polyenes.

\section{References}

Ghosh A K, Morel D L, Feng T, Shaw R F and Rowe C A Jr 1974 J. Appl. Phys. 45230

Glenis S, Tourillon G and Garnier F 1984 Thin Solid Films 1229

Hoshino Y and Tateishi K 1979 J. Phys. Soc. Jpn. 4672

Lampert M A 1964 Rep. Prog. Phys. 27329

Mallick B, Ghosh A and Misra T N 1979 Bull. Chem. Soc. Jpn. 522091

Pal P, Ghosh D and Misra T N 1988 J. Phys. Soc. Jpn. 571006

Pal P and Misra T N 1989 J. Phys. D: Appl. Phys. 221358

Pal P and Misra T N 1993 Mol. Cryst. Liq. Cryst. 230649

Roberts G G and Schmidt F W 1969 Phys. Rev. 180785

Rose A 1955 Phys. Rev. 971938

Rose A 1963 Concepts in photoconductivity and allied problems (New York: Interscience)

Weast R C, Astle M J and Beyer W H 1986 CRC handbook of chemistry and physics (Boca Ralton, Florida: CRC Press, Inc.) 67th ed. p. E-53

Yasuhara A, Akiyama S and Nakagawa M 1972 Bull. Chem. Soc. Jpn. 453638 